\title{
A enfermagem no cuidado com o idoso portador de alzheimer
}

\author{
Nursing care for the older alzheimer carrier
}

\section{La enfermería en el cuidado con el idoso portador de alzheimer}

\begin{abstract}
José Nilton Ferreira Sales ${ }^{1}$, Kátia Maria Azevedo de Oliveira Santos ${ }^{1}$, Raimundo Nonato Cardoso Miranda $^{2}$, Marcos Antônio Sousa da Silva ${ }^{1 *}$, Elielton da Silva Batista ${ }^{1}$, Jucileia Ramos da Silva ${ }^{1}$, Jane Sousa Silva ${ }^{1}$, Janaina Sousa Silva ${ }^{1}$, José Diego da Silva Boaventura ${ }^{1}$, Jociane Cardoso Santos Ferreira $^{1}$, João Rodrigues dos Santos Neto ${ }^{1}$, Marcilene dos Santos da Silva ${ }^{1}$, Jozenilde de Souza Silva ${ }^{1}$, Karllieny de Oliveira Saraiva ${ }^{1}$, Kivia Késsia Moura de Abreu¹.
\end{abstract}

\section{RESUMO}

Objetivo: Elencar na literatura evidências cientificas acerca dos cuidados de Enfermagem ao Idoso portador de Alzheimer. Metodologia: Trata-se de uma revisão bibliográfica, integrativa, descritiva com abordagem qualitativa, na qual consultou-se por meio de descritores as bases de dados BVS (Biblioteca Virtual em Saude), Lilacs e Medline. Resultado: Analisou-se 13 artigos que abordaram cuidados de Enfermagem ao Idoso portador de Alzheimer, na qual observou-se que os profissionais de enfermagem que atuam na gestão do cuidado a essa clientela devem criar métodos interativos com o paciente e os familiares, objetivando desenvolver conhecimento específico e consciência ampliada em relação às heterogeneidades do processo natural do envelhecimento, distinguindo-o do estado patológico, elaborando e promovendo uma assistência de qualidade e cuidado integral à saúde dos idosos. Conclusão: Evidenciou-se que o enfermeiro tem o papel fundamental na orientação e cuidados de enfermagem ao paciente e sua família, desde o diagnóstico ao estágio mais grave.

Palavras chaves: Enfermagem, Cuidado com o idoso, Alzheimer.

\begin{abstract}
Objective: To publish scientific literature about two nursing care for Alzheimer's disease. Methodology: This is a bibliographic review, integrative, descriptive with qualitative approach, which is consulted by descriptors as databases of VHL (Virtual Library in Saude), Lilacs and Medline. Result: Analysis of 13 articles addressing the care of patients with Alzheimer's Disease, who observed that they were nursing professionals who wanted to care for this clientele to create interactive methods with family patients, aiming to develop specific confecimento and extended consciência em relação às heterogeneities of natural process of envelhecimento, distinguishing-o of pathological state, elaborating and promoting a assistência de qualidade and integral care à saúde dos idosos. Conclusion: Evidenciou-se that or nurse tem or fundamental role in the orientation of nursing care to patient and his family, from or diagnosis to more serious stagio.
\end{abstract}

Palavras chaves: Nursing, Care as good, Alzheimer's.

\section{RESUMEN}

Objetivo: El contenido de la literatura científica científica acerca de los cuidados de la enfermedad en el puerto de Alzheimer. Metodología: Trata-se de uma revisión bibliográfica, integrativa, descrita com abordagem qualitativa, na qual consultou-se para meio de descritores como bases de dados BVS (Biblioteca Virtual en Saude), Lilacs y Medline. Resultado: Análisis 13 artículos que abordan el cuidado de la enfermedad en el puerto de Alzheimer, en el cual se observan las profesiones de enfermería y el manejo del cuidado de la salud y el cuidado de los pacientes. conhecimento específico y consciência ampliada en relación con las heterogeneidades del proceso natural, la distinción, el estado patológico, el desarrollo y la promoción de la calidad y el cuidado integral de la salud y la salud. Conclusión: Evidencia de la enfermedad o el papel fundamental en la orientación y los cuidados de la salud en el paciente y en la familia, desde el momento en que se informa sobre la situación de la tumba.

Palavras chaves: Enfermagem, Cuidado con el idoso, Alzheimer.

\footnotetext{
${ }^{1}$ Faculdade de Ciência e Tecnologia do Maranhão (FACEMA).

2 Docente da FACEMA. *E-mail: jrfarmaceutico@hotmail.com
}

SUBMETIDO EM: 8/2018 


\section{INTRODUÇÃO}

O Alzheimer é uma doença neurodegenerativa de caráter progressivo e letal que se manifesta por degradação cognitiva da memória, comprometendo progressivamente as atividades da vida diária e apresentando uma variedade de sintomas neuropsiquiátricos e de alterações no comportamento do indivíduo (TALMELLI et a., 2013).

Em estágios iniciais da doença, podem ser observadas a perda de memória episódica e a dificuldade na aquisição de novas tarefas. Esses danos gradualmente envolvem outros comprometimentos cognitivos, como a capacidade de julgamento, cálculo, capacidade de abstração e habilidades viso espaciais. A afasia pode ocorrer na fase intermediária, com dificuldade em nomear objetos ou escolher a palavra certa para expressar ideias, assim como apraxia. Em estágios terminais da doença, mudanças notáveis são observadas, desde alteração do ciclo do sono, alterações comportamentais (irritação e agressividade), sintomas psicóticos, até inabilidade para caminhar, falar e realizar o autocuidado. (ZIDAN et al., 2012).

A idade (envelhecimento) é o fator de risco mais conhecido e importante para a forma o desenvolvimento da doença de Alzheimer. No entanto existe outros fatores de risco, tais como: medicamentos diversos, trauma craniano, exposição à radiação, estilo de vida, estresse, infecções, doenças imunológicas, câncer, altos níveis de colesterol e de homocisteína, a obesidade, diabetes (Santos et al., 2015).

A incidência global de demência vem aumentando de maneira drástica nas últimas décadas. Em 2012 as estimativas indicaram cerca de 7,7/1000 pessoas por ano, isso se traduz em cerca de 1 novo caso a cada 4 segundos, já em 2015 estimou-se que cerca de 7,5/1000 pessoas por ano, ou equivale a um novo caso a cada 7 segundos (SILVA, 2012).

Nessa perspectiva no estudo de meta-analise de Santana e colaboradores (2015) demostrou que prevalência dessa doença acima de 60 anos de idade varia de 5 a $7 \%$ sendo que nos países da América latina foi de $8,5 \%$ e nos mais países da África Sub-Sariana foi de 2 a $4 \%$.

No entanto estudos recentes indicam uma estabilização ou até mesmo a redução na incidência de demência nos países desenvolvidos, associando-se à melhora do estilo de vida e consequentemente ao controle dos fatores de risco o que levará também a uma diminuição da mortalidade da doença (SANTANA et al., 2015).

Com o crescente aumento da longevidade, a enfermagem suscitou a precisão de se adquirir mais informações a respeito dessa população, no intuito de desenvolver estudos que venham ajudar nos cuidados eficazes aos idosos, no anseio de estabelecer a saúde ou somente garantir o prolongamento de um envelhecimento proveitoso e com qualidade de vida (CARVALHO, 2008).

Os cuidados prestados devem ser tanto de caráter físico quanto emocional, porque dessa forma o cuidador poderá evitar problemas graves que venha a envolver o idoso, e com isso ajuda-lo nas execuções das atividades que já estão modificadas (CARVALHO, 2008). Diante disso, o presente estudo tem como objetivo realizar uma revisão da literatura acerca dos cuidados de Enfermagem ao Idoso portador de Alzheimer.

\section{METODOLOGIA}

Esta pesquisa foi caracterizada por ser bibliográfica, integrativa, descritiva com abordagem qualitativa na qual se empregará uma revisão integrativa da literatura. Este procedimento foi escolhido por possibilitar a síntese e analise do conhecimento científico já produzido sobre o tema "A enfermagem no cuidado ao idoso portador de Alzheimer". 
A pesquisa bibliográfica foi conceituada como um procedimento formal com método de pensamento reflexivo que propõe um tratamento cientifico e se estabelece no caminho para se conhecer a realidade ou para descobrir verdades parciais (MARCONI et al., 2012). Desta forma o estudo proposto foi formado com o apoio em material já produzido. Visto a obrigatoriedade de alguns trabalhos ser valer unicamente de fontes bibliográficas (GIL; 2007)

Para a identificação do tema e seleção da questão de pesquisa utilizou-se nas bases de dados a seguinte questão: "Quais evidencias cientifica acerca dos cuidados de enfermagem ao idoso portador de Alzheimer?", a partir disto elaborou-se a seguinte estratégia de busca. Identificou-se com DECS o total de 04 palavras sendo elas: enfermagem, cuidado, idoso, Alzheimer. Para seleção das fontes, utilizou-se a procura pelas palavras chave como uso do AND, para ajudar a encontrar os artigos da base de dado online. As bases de dados escolhidas foram Biblioteca Virtual em Saúde (BVS) com colaborador da Rede Lilacs e Medline.

Como critério de inclusão foi utilizado os artigos completos em língua portuguesa e espanhola, publicados a partir de 2005 até Dezembro de 2016, aproveitando-se artigos com abordagem qualitativa, descritiva, reflexivo e relato de experiência, disponíveis eletronicamente, que tratem diretamente da enfermagem no cuidado ao idoso portador de Alzheimer.

Como critérios de exclusão, serão excluídas teses, monografias, resumos, dissertações e artigos que não atenderam a temática em estudos.

Para realização da categorização dos estudos selecionados, foi utilizado um instrumento denominado de matriz de síntese ou matriz de análise, que permite analisar separadamente cada artigo, extrair e organizar os dados tanto num nível metodológico quanto em relação aos resultados das pesquisas.

\section{RESULTADOS}

Para análise dos dados documentais dos 13 artigos utilizados no preste estudo, foram utilizados cálculos matemáticos e inferência, que são apresentados em tabelas, figuras e quadros para melhor compreensão.

A Tabela 1 mostra que, dos anos de publicação dos artigos, os de 2007, 2008, 2009, 2010, 2011, 2012 e 2015 tiveram maior prevalência, com $15,4 \%$, seguido do ano de 2014 , com $7,6 \%$. Comparado a isso, os anos de 2005, 2006, 2013 e 2016, com 0,0\%, foram os de menor número de artigos publicados. De acordo com a tabela, percebe-se que o estudo obteve um intervalo de tempo adequado acerca do tema, possibilitando um contraste e comparação dos anos.

Tabela 1: Distribuição dos estudos incluídos na amostra, referentes ao ano de publicação

\begin{tabular}{ccc}
\hline ANO DE PUBLICAÇÃO & NUMERO ABSOLUTO & PERCENTUAL \\
\hline 2005 & 0 & $0 \%$ \\
2006 & 0 & $0 \%$ \\
2007 & 2 & $15,4 \%$ \\
2008 & 2 & $15,4 \%$ \\
2009 & 2 & $15,4 \%$ \\
2010 & 2 & $15,4 \%$ \\
2011 & 2 & $15,4 \%$ \\
2012 & 2 & $15,4 \%$ \\
2013 & 0 & $0 \%$ \\
2014 & 1 & $7,6 \%$ \\
2015 & 2 & $15,4 \%$ \\
2016 & 0 & $0 \%$ \\
\hline TOTAL & 13 & $100 \%$
\end{tabular}

Fonte: Pesquisa realizada em bancos em dados online coletados no ano de 2016. 
Na Tabela 2 fica evidenciado que a fonte online LILACS (100\%) disponibilizou mais estudos a respeito do idoso portador de Alzheimer e teve maior relevância no estudo, em comparação com a fonte MEDLINE $(0,0)$.

Tabela 2: Distribuição dos estudos incluídos na amostra, referentes à fonte online de publicação

\begin{tabular}{lcc}
\hline FONTE ONLINE & NUMERO ABSOLUTO & PORCENTAGEM \\
\hline LILACS & 13 & $100 \%$ \\
MEDLINE & 0 & $0 \%$ \\
\hline TOTAL & 13 & $100 \%$
\end{tabular}

Fonte: Pesquisa realizada em bancos de dados online coletados no ano de 2016.

Na Tabela 3, observa-se que a Revista Kairós teve maior número de artigos publicados, com 15,4\%. De um total de 11 de revista, houve, apenas um artigo de cada, utilizados no estudo, que somados tiveram grande êxito para o trabalho.

\begin{tabular}{lcc} 
Tabela 3: Distribuição dos estudos incluídos na amostra, referentes ao nome do período \\
\hline NOME DO PERIÓDICO & NUMERO ABSOLUTO & PORCENTAGEM \\
\hline Rev. Kairós & 2 & $15,4 \%$ \\
Ciênc. cuid. saúde & 1 & $7,6 \%$ \\
Cogitare enferm & 1 & $7,6 \%$ \\
Estud. interdiscip. & 1 & $7,6 \%$ \\
envelhec & 1 & \\
Mundo saúde (Impr) & 1 & $7,6 \%$ \\
Psico. (Porto Alegre) & 1 & \\
Rev. Esc enferm USP & 1 & $7,6 \%$ \\
Rev. eletrônica enferm & 1 & $7,6 \%$ \\
Rev. enferm UERJ & 1 & $7,6 \%$ \\
Texto contextos & & $7,6 \%$ \\
(Porto Alegre) & 1 & \\
Rev. Méd. Clín. Condes & 1 & $7,6 \%$ \\
Rev. latinoam. Bioét & 13 & $7,6 \%$ \\
\hline \multicolumn{1}{c}{ TOTAL } & $100 \%$
\end{tabular}

Fonte: Pesquisa realizada em bancos de dados online coletados no ano de 2016.

O Quadro 1 mostra que, do total de estudos incluídos neste trabalho, o idioma Português $(n=11)$ teve maior prevalência com 84,6\%; comparados ao idioma Espanhol $(n=2)$, com $15,4 \%$, para realizações dos estudos, e/ou considera como grande interesse dos estudantes e profissionais de saúde pelo tema. Vale ressaltar que o idioma Espanhol foi de suma importância na obtenção dos dados e propiciou uma contribuição significável para este estudo. 
Quadro 1: Distribuição sinóptico demonstrativo quanto ao delineamento do estudo, tipo de abordagem, objetivo do estudo

\begin{tabular}{|c|c|c|c|}
\hline 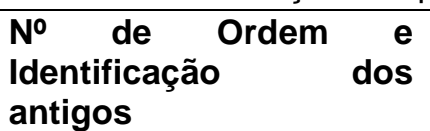 & $\begin{array}{l}\text { Tipo de estudo ou } \\
\text { delineamento }\end{array}$ & Tipo de abordagem & Objetivo do estudo \\
\hline $\begin{array}{l}\text { A1 } \\
\text { BAGNE, } \\
\begin{array}{l}\text { GASPARINO, } \\
(2014)\end{array}\end{array}$ & Quantitativo & Medidas profiláticas & $\begin{array}{l}\text { Avaliar a qualidade de vida do cuidador do portador da Doença } \\
\text { de } \\
\text { e sua relação comeimer o nível de independência funcional do } \\
\text { portador. }\end{array}$ \\
\hline $\begin{array}{lrr}\text { A2 } & \\
\text { ARAÚJO, } & \text { C. } \quad \text { L. } & \text { O.; } \\
\text { OLIVEIRA, } & \text { J. } & \text { F.; } \\
\text { PEREIRA J. M., (2012) }\end{array}$ & Qualitativo-quantitativo & $\begin{array}{l}\text { Perfil e conhecimento dos } \\
\text { cuidadores de portadores da } \\
\text { DA }\end{array}$ & $\begin{array}{l}\text { Mapear o perfil sociodemográfico; como: faixa etária; sexo; } \\
\text { escolaridade; grau de parentesco com o idoso. }\end{array}$ \\
\hline $\begin{array}{l}\text { A3 } \\
\text { SEIMA, } \quad \text { M. } \quad \text { D.;; } \\
\text { LENARDT, M. H., (2011) }\end{array}$ & De corte transversal & $\begin{array}{l}\text { Perfil dos cuidadores dos } \\
\text { portadores da DA e medidas } \\
\text { profiláticas }\end{array}$ & $\begin{array}{lllll}\text { Foi identificar o nível } & \text { de } & \text { sobrecarga } & \text { do } \\
\text { cuidador familiar do idoso com Alzheimer } & \text { de uma comunidade. }\end{array}$ \\
\hline $\begin{array}{l}\text { A4 } \\
\text { VALENTINI, I. } \\
\text { ZIMMERMANN, } \\
\text { FONSECA R. P., (2010) }\end{array}$ & $\begin{array}{l}\text { Quantitativa descritiva } \\
\text { e qualitativa }\end{array}$ & Diagnostico & $\begin{array}{l}\text { Foi } \quad \text { investigar } \\
\text { ocorrência de depressão e de ansiedade em cuidadores } \\
\text { primários } \\
\text { de adultos idosos portadores de Demência Tipo Alzheimer. }\end{array}$ \\
\hline $\begin{array}{l}\text { A5 } \\
\text { VALIM, M. D., et al., } \\
(2010)\end{array}$ & Estudo de caso & $\begin{array}{l}\text { Perfil dos cuidadores dos } \\
\text { portadores da DA }\end{array}$ & $\begin{array}{l}\text { Compreender o significado atribuído pelo familiar na prestação } \\
\text { do cuidado domiciliar a um paciente portador de DA. }\end{array}$ \\
\hline \begin{tabular}{lll}
\multicolumn{3}{c}{ A6 } \\
SANTANA, & R. & F.; \\
ALMEIDA, & K, & S.; \\
SAVOLDI, N, & A, & M.; \\
$(2007)$ & & \\
\end{tabular} & $\begin{array}{l}\text { Qualitativo, tipo estudo } \\
\text { de caso, descritivo- } \\
\text { exploratório, }\end{array}$ & $\begin{array}{l}\text { Perfil dos cuidadores dos } \\
\text { portadores da DA e medidas } \\
\text { profiláticas }\end{array}$ & $\begin{array}{l}\text { Descrever a orientação recebida pelo cuidado do portado de } \\
\text { Alzheimer antes de sua participação no Pólo de Neurogeriatria. }\end{array}$ \\
\hline $\begin{array}{l}\text { A7 } \\
\text { DOMINGUES, M. A. R. C.; } \\
\text { SANTOS, } \quad \text { C. } \quad \text { F.; } \\
\text { QUINTANS, J. R., (2009) }\end{array}$ & $\begin{array}{l}\text { Estudo retrospectivo, } \\
\text { quantitativo, } \\
\text { exploratório, } \\
\text { não probabilístico. }\end{array}$ & $\begin{array}{l}\text { Perfil dos usuários e mediadas } \\
\text { profiláticas }\end{array}$ & $\begin{array}{l}\text { Foi apresentar o perfil dos usuários que utilizavam o serviço de } \\
\text { apoio telefônico da da ABRAz } \\
\text { Associação Brasileira de Alzheimer e propor estratégias para } \\
\text { responder à demanda dos usuários desse serviço. }\end{array}$ \\
\hline $\begin{array}{l}\text { A8 } \\
\text { GUTIERREZ, B. A. O.; } \\
\text { SILVA, H. S. (2008) }\end{array}$ & Tipo estudo de caso & $\begin{array}{l}\text { Fatores de risco para uma } \\
\text { paciente portadora da DTA }\end{array}$ & 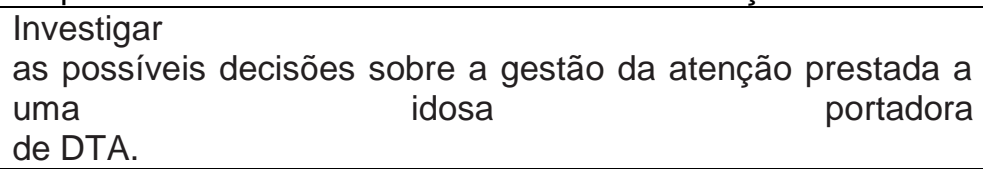 \\
\hline
\end{tabular}

REAS/EJCH | Vol.Sup.18 | e235 | DOI: https://doi.org/10.25248/reas.e235.2019 Página 5 de 9 


\begin{tabular}{|c|c|c|c|}
\hline $\begin{array}{llll}\text { A9 } & & & \\
\text { ARRUDA, } & \text { M. } & \text { C.; } \\
\text { ALVAREZ, } & \hat{A} . & \text { M.; } \\
\text { GONÇALVE, } & \text { L. } & \text { H. } & \text { T., } \\
(2008) & & & \\
\end{array}$ & $\begin{array}{l}\text { Abordagem } \\
\text { exploratório-descritiva }\end{array}$ & $\begin{array}{l}\text { Perfil dos cuidadores dos } \\
\text { portadores da DA e medidas } \\
\text { profiláticas }\end{array}$ & $\begin{array}{l}\text { Identificar as características do familiar cuidador de portador da } \\
\text { Doença de Alzheimer (DA). }\end{array}$ \\
\hline $\begin{array}{l}\text { A10 } \\
\text { LIMA, } \\
\text { MARQUES, J. C., (2007) }\end{array}$ & Não diz & Medidas profiláticas & $\begin{array}{l}\text { Apresentar algumas considerações que podem ser úteis para } \\
\text { a compreensão das relações interpessoais que ocorrem nas } \\
\text { famílias de portadores de Doença de Alzheimer. }\end{array}$ \\
\hline $\begin{array}{l}\text { A11 } \\
\text { CELICH, } \quad \text { K. L. S.; } \\
\text { BATISTELLA, M., (2007) }\end{array}$ & $\begin{array}{l}\text { Qualitativa } \quad \text { com } \\
\text { abordagem } \\
\text { fenomenológica }\end{array}$ & $\begin{array}{l}\text { Perfil dos cuidadores dos } \\
\text { portadores da DA e medidas } \\
\text { profiláticas }\end{array}$ & $\begin{array}{l}\text { Descrever as vivências e os sentimentos de ser um cuidador } \\
\text { familiar do portador da } \\
\text { Alzheimer (DA). }\end{array}$ \\
\hline $\begin{array}{l}\text { A12 } \\
\text { ESPÍNOLA, H. R. G., } \\
(2012)\end{array}$ & Retrospectivo & $\begin{array}{l}\text { Perfil dos cuidadores dos } \\
\text { portadores da DA e medidas } \\
\text { profiláticas }\end{array}$ & $\begin{array}{l}\text { Fornecer ferramentas que são } \\
\text { útil para os médicos que atendem idosos. }\end{array}$ \\
\hline $\begin{array}{c}\text { A13 } \\
\text { COÊLHO, A. D. A., (2012) }\end{array}$ & $\begin{array}{l}\text { Transversal } \\
\text { descritivo }\end{array}$ & $\begin{array}{l}\text { Prevalência de ulcera em } \\
\text { idosos }\end{array}$ & $\begin{array}{l}\text { Estimar a prevalência de úlcera por pressão num serviço de } \\
\text { atendimento domicilia. }\end{array}$ \\
\hline
\end{tabular}

Fonte: Elaboração própria. 


\section{DISCUSSÃO}

O aumento dos índices do mal de Alzheimer tem gerado uma necessidade de se obter um conhecimento maior para uma melhor assistência para esses pacientes. Cabe a enfermagem atividade de prevenção, inclusão, baseado no processo de humanização onde analise o cliente como um todo, não focando somente a patologia, mas visando valores, princípios, ideias e atitudes, proporcionando uma melhora na qualidade de vida desses portadores de Alzheimer.

\section{As orientações desenvolvidas por profissionais enfermeiros à cuidadores e os cuidados prestado a pessoa portadora de alzheimer}

No que concerne as orientações e cuidados prestado a cuidadores de idosos acometido pelo Alzheimer, é de grande responsabilidade dos profissionais área da saúde em especial a enfermagem tem como uma de suas características principais a educação em saúde, acerca de como conduzias a aliança entre orientação, cuidadores e idoso demenciados (ESPÍNOLA et al., 2012).

Desempenhar atividades em grupo tem se tornado uma ponte fundamental no ato de orientar o cuidado, onde as elaborações dessas atividades são desenvolvidas em conjunto, independente da fase em que se encontra a doença. Com isso as atividades desenvolvidas se alternam em duas, os grupos de orientação e suporte estimulando onde os encontros servem como meio de socializar os convívios e vivencias além de fortalecer os saberes e o aprendizado recíproco do grupo para que assim possam desempenhar adaptações a sua realidade. $O$ outro objetiva o fortalecimento da autoestima dessas pessoas que desempenham atividade de cuidadores e, especialmente para resgatar a precisão de tomar cuidado de si para que dessa forma possam cuidar do outro (SANTANA et al.,2007, ARRUDA et al., 2008).

No que se refere a assistência realizada ao idoso, e quando se sugere uma intervenção, a equipe interprofissional necessita se assegurar do número e das características das enfermidades, pois o paciente e familiares devem ser informados acerca da etiologia das enfermidades que acometem o paciente, bem como o tratamento. Desta forma, eles serão instruídos como as atividades de cuidado serão desenvolvidas visando a atenção e os prováveis agravos em relação aos procedimentos que podem causar. E com isso seja possível se definir as mais variadas formas de assistência ao paciente idoso demenciados é onde o desenvolver dessas tarefas exijam mais dos profissionais uma abrangência das metas e da relação entre maleficio e benefício dos procedimentos desenvolvidos (GUTIERREZ et al., 2008; SEIMA et al., 2011).

Importância da elaboração dos diagnósticos enfermagem em idosos com
doença de alzheimer

Uma das atividades da enfermagem é a de poder associar na elaboração de diagnósticos os principais cuidados que o paciente necessita, que pode vir a somar junto à ação de outros profissionais em especial os neurologistas na produção de planos assistências com a intenção de promover o bem-estar de pacientes idosos demenciados (BAGNE et al., 2014).

Esses diagnósticos quando realizado permite o levantamento de dados e investigações eficazes e resultados positivo para o desenvolvimento de uma assistência de enfermagem com qualidade para o idoso acometido pela doença de Alzheimer cooperando com outros profissionais a confirmá-lo, delimitando ou refutando alguns outros possíveis diagnósticos. E dessa maneira torna-se imprescindível a participação interdisciplinar entre os profissionais que desempenham a arte do cuidar de pessoas idosas demenciados (COÊLHO et al., 2012).

\section{Os cuidados de enfermagem realizados à pessoa idosa com doença de alzheimer e seus cuidadores}

O enfermeiro é o profissional que por excelência mantem contato direto com pacientes seja em qualquer tipo de enfermidade que ele apresente, e no que concerne à tarefa de realizar o cuidado é um dos profissionais com capacidade para desenvolver tal tarefa (CELICH et al., 2007). 
Constituindo-se de capacidades empíricas de conhecimentos o profissional de enfermagem, dentro de suas atribuições técnicas cientificas poderá desenvolver estimulo da função cognitiva diminuindo dessa maneira a ansiedade e agitação, garantindo e promovendo a segurança física, aprimorando a comunicação e promovendo o bem-estar do indivíduo no que diz respeito às suas atividades de autocuidado, procurando suprimir às necessidades de sociabilidade, solicitando a atividade de repouso equilibrados e elaborando um plano nutricional adequado (SANTOS et a., 2009, DOMINGUES et al., 2009).

A participação de profissionais enfermeiros na realização de intervenções com pessoas que desempenham atividades de cuidadores, de longa duração, tem como objetivo principal desenvolver a proteção contra sintomas depressivos. Os cuidadores que através das intervenções venham a adquirir conhecimentos e desenvolver habilidades, e assim utilizar esses recursos para poder desenvolver uma assistência mais ativa quanto a realização do cuidado, mas também para desenvolver mecanismos de enfrentamentos e os desafios de lidar com a morte de um ente valioso (ARAÚJO et al., 2012).

A ação desenvolvida pela enfermagem tem uma intensa atividade no auxílio prestado ao cuidador assim como as intervenções que são realizadas para o cliente com doença de Alzheimer (VALIM et al., 2010).

Por conseguinte, na perspectiva assistencial a enfermagem, é caracterizada como a profissão da área da saúde que tem como vertente o saber técnico, cientifico, ético e cultural. O profissional enfermeiro está inteiramente comprometido, para auxiliar o indivíduo e seus familiares a buscar meios de compreender e encarar seu conhecimento acerca da doença ou sofrimento e, dessa forma contribuir para que eles encontrem meios de cuidar ou se necessário, descobrirem novos significados para essa experiência (LIMA et al., 2007; VALENTINI et al., 2010).

\section{CONSIDERAÇÕES FINAIS}

O envelhecer traz consigo complicações de saúde, dentre elas a doença de Alzheimer, doença sem cura e seu tratamento consiste em tentar controlar o déficit de memória e outras complicações. Assim é imprescindível um cuidado especial, sendo a equipe de enfermagem fundamental no desenvolvimento de uma assistência de qualidade.

Este estudo permitiu discutir os cuidados de Enfermagem ao Idoso portador de Alzheimer, segundo a literatura científica. Concluiu-se então, que o enfermeiro tem o papel fundamental na orientação e cuidados de enfermagem ao paciente e sua família, desde o diagnóstico ao estágio mais grave. Para isso é importante possuir conhecimentos, habilidades, técnicas e humanização para o manejo dos casos. O resultado do presente estudo poderá colaborar para que os profissionais da enfermagem tenham mais conhecimento quanto a importância da assistência de enfermagem ao paciente portador de Alzheimer.

\section{EFERÊNCIAS}

1. VALENTINI, IB et al. Ocorrência de depressão e ansiedade em cuidadores primários de indivíduos com demência tipo Alzheimer: estudos de casos. Estud. interdiscipl. envelhec., Porto Alegre, v. 15, n. 2, p. 197 217, 2010.

2. SANTANA, RF et al. indicativos de aplicabilidade das orientações de enfermagem no cotidiano de cuidadores de portadores de Alzheimer. Rev. Esc. Enfern USP 2009, 43(2):459-64.

3. MARCONI, MA et al. Metodologia do trabalho científico. São Paulo, 2012.

4. LIMA, LD et al. Relações interpessoais em famílias com portador da doença de Alzheimer. PUCRS. Psico, Volume 38, Number 2, 2007, pp. 157-165(9).

5. GIL, A. C. Como elaborar projeto de pesquisa. São Paulo, 2007.

6. GUTIERREZ, BAO et al. Dilemas bioéticos na assistência prestada ao idoso portador de demência do tipo Alzheimer. revista Kairós, São Paulo, 11(2), dez. 2008, pp. 159-180.

7. BAGNEI, BM et al. Qualidade de vida do cuidador do portador de doença de Alzheimer. Rev enferm UERJ, Rio de Janeiro, 2014 mar/abr; 22(2):258-63. 
8. ARAÚJO, CLO et al. Perfil de cuidadores de idosos com doença de Alzheimer. Revista Kairós Gerontologia, São Paulo (SP), Brasil,15(2), março 2012: 119-137. TALMELLI, LFS al et. Doença de Alzheimer: declínio funcional e estágio da demência. Acta Paul Enferm. 2013; 26(3):219-25.

9. ZIDAN, M etal. Alterações motoras e funcionais em diferentes estágios da doença de Alzheimer. Rev Psiq Clín. 2012;39(5):161-5.

10. SILVA TS. Diagnóstico da Doença de Alzheimer na População Brasileira: um Levantamento Bibliográfico. Revista Psicologia e Saúde, v. 4, n. 2, jul./dez. 2012.

11. SANTOS, MD et al. Percepção da funcionalidade nas fases leve e moderada da doença de Alzheimer: visão do paciente e seu cuidador. Rev. Bras. Geriatr. Gerontol., Rio de Janeiro, 2015; 18(2):339-349.

12. CARVALHO, IC et al. Convivendo com o portador de Alzheimer: perspectivas do familiar cuidad or. Rev Bras Enferm, Brasília 2008 Bras Enferm, Brasília 2008 jul-ago; 61 (4): 508-13.

13. SEIMA, MD et al. A condição de saúde e satisfação com a vida do cuidador familiar de idoso com Alzheimer. Colombia Médica. Vol. 42 № 2 (Supl 1), 2011 (Abril-Junio).

14. VALIM, MD et al. A doença de Alzheimer na visão do cuidador: um estudo de caso. Rev. Eletr. Enf. [Internet]. 2010;12(3):528-34.

15. DOMINGUES, MARC et al. Doença de Alzheimer: o perfil dos cuidadores que utilizam o serviço de apoio telefônico da ABRAz - Associação Brasileira de Alzheimer. O Mundo da Saúde, São Paulo: 2009;33(1):161169.

16. ARRUDA, MC et al. O familiar cuidador de portador de doença de alzheimer participante de um grupo de ajuda mútua. Cienc Cuid Saude, 2008 Jul/Set; 7(3):339-345.

17. CELICH, KLS et al. Ser cuidador familiar do portador de doença de Alzheimer: vivências e sentimentos desvelados. Cienc Cuid Saude, 2007 Jul/Set; 10(1):120-201.

18. ESPÍNOLA, HRG et al. Implicações da doença de Alzheimer na qualidade de vida do cuidador: um estudo comparativo. Cad. Saúde Pública, Rio de Janeiro, 26(5):891-899, mai, 2012.

19. COÊLHO, ADA et al. O Idoso e a úlcera por pressão em serviço de atendimento domiciliar. Rev Rene. 2012; 13(3):639-49. 